\title{
Structural Characterization of Carbon Nanotubes
}

Ph. Lambin, ${ }^{*}$ J.F. Colomer, ${ }^{*}$ L. Henrard, ${ }^{*}$ A. Lucas, ${ }^{*}$ and V. Meunier ${ }^{* *}$

* Laboratoire de Physique du Solide, Facultés Universitaires Notre-Dame de la Paix, 61 Rue de Bruxelles, B5000 Namur, Belgium

** Computer Science and Mathematics Division, Oak Ridge National Laboratory, Oak Ridge, TN 37831, USA

Carbon nanotubes are long, hollow cylindrical molecules discovered fifteen years ago. The structure of a single-wall nanotube, made of just one graphene layer, is conventionally defined by a pair of integer numbers, $n$ and $m$, corresponding to the circumferential vector when the cylinder is developed on a plane. Most of the properties of a single-wall nanotube depend on its diameter and, to a lesser extent, on the helicity of its atomic structure. The helicity is the angle that the circumference makes with the closest zig-zag chain of $\mathrm{C}$ in the planar development. The diameter and helicity of a nanotube completely define the pair of wrapping indices $(n, m)$ that determine its atomic structure.

These two parameters are difficult to measure experimentally. ${ }^{1}$ In the case of an individual nanotube, the most powerful techniques giving access to both diameter and helicity are STM and selected-area electron diffraction. As it is detailed in the paper, the interpretation of the STM images of a nanostructure is more complicated than in the case of a planar crystalline surface, due to quantum effects that distort the image which the microscope gives of the sample. Also in electron diffraction, the curvature of the atomic structure of a nanotube has a strong influence on the diffraction it produces. Due to all these effects, the analysis of experimental diffraction patterns and STM images of a nanotube is greatly improved by comparison with computer-generated data.

As an example, fig. 1(a) is an experimental electron diffraction pattern of a small bundle of singlewall nanotubes produced by catalytic chemical vapour deposition, which demonstrates that all the nanotubes have the same diameter and helicity. The intensity profile measured along the equatorial line is shown in fig. 1 (b). It reveals Bragg peaks generated by the close-packed array of tubes in the bundle. Fig. 1(c) is the intensity profile computed for a bundle made of all $(14,5)$ nanotubes, with the horizontal line representing a possible saturation level of the experimental intensities. The calculation of the diffraction intensity is based on the kinematical theory, which produces reliable data for carbon nanotubes. ${ }^{2}$

In this paper, STM topographical images of the nanotubes are calculated in tight-binding approximation, using first-order perturbation theory of the tunnelling current. ${ }^{3}$ As an illustration, fig. 2 shows STM images computed for two semiconductor nanotubes, $(13,6)$ and $(14,6)$, which have nearly the same atomic structure. However, their STM images look different. In fact, the image of $(13,6)$ computed for a positive tip potential resembles closely that of $(14,6)$ obtained with a negative bias, and vice versa. This is so, because a positive STM tip probes the occupied electronic states of the sample, whereas a negative tip probes the unoccupied states. On going from the $(13,6)$ 
nanotube to $(14,6)$, one chiefly exchanges the wave-function characteristics of the highest occupied states with those of the lowest unoccupied states. Fig. 2 is an example, among others, which demonstrates that STM mixes both structural and electronic properties of the carbon nanotubes.
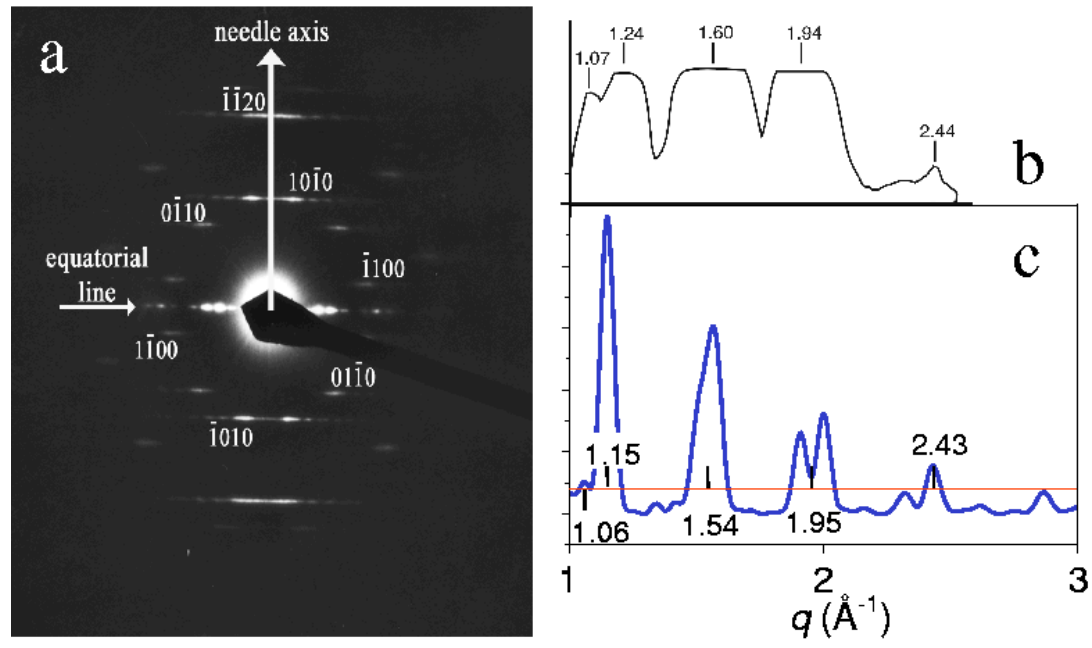

Fig. 1. (a) Experimental diffraction pattern of a bundle of single-wall nanotubes. (b) Intensity measured along the equatorial line. (c) Intensity computed for a bundle of $(14,5)$ nanotubes.
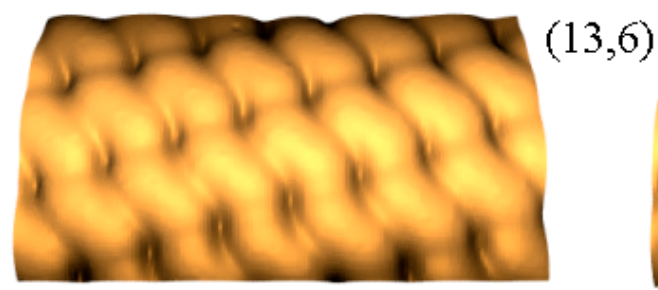

$$
V=-0.4 \mathrm{~V}
$$
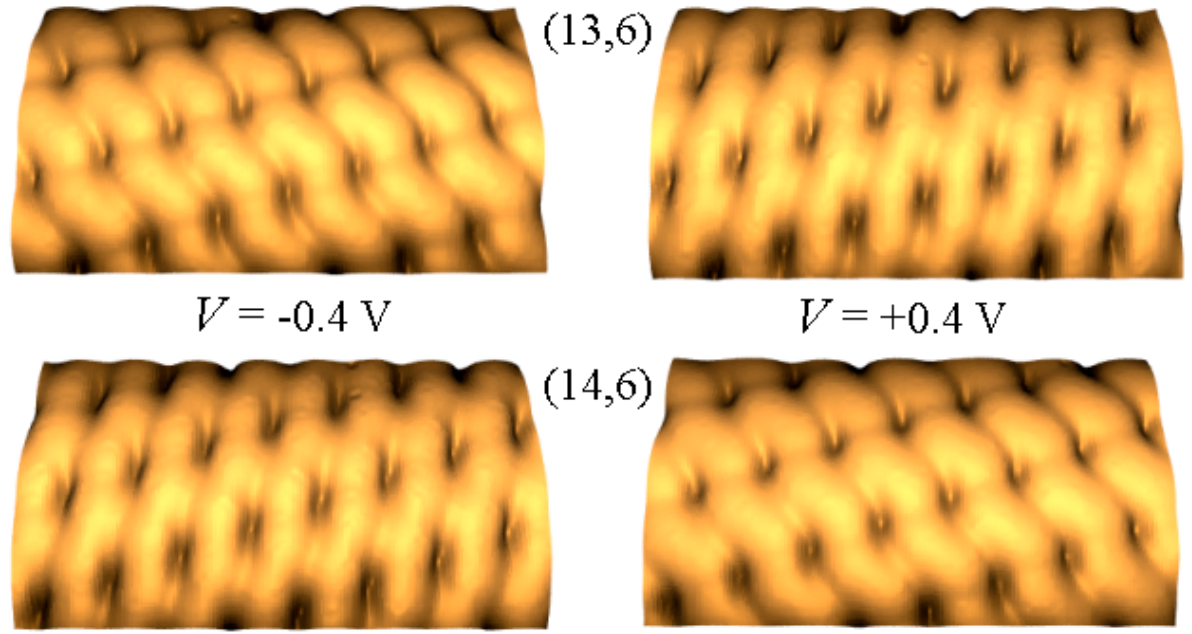

$$
V=+0.4 \mathrm{~V}
$$

$(14,6)$

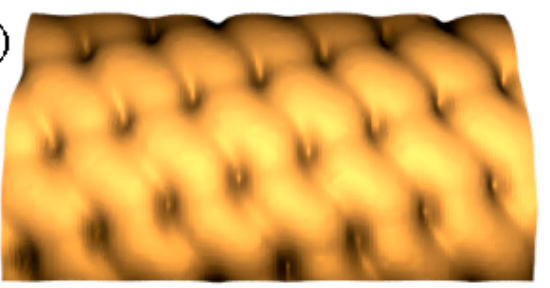

Fig. 2. STM images of the semiconductor $(13,6)$ and $(14,6)$ nanotubes, for two bias potentials $\mathrm{V}$ of the tip, probing the unoccupied (left) and occupied electronic states (right) of the sample.

1 Ph. Lambin, A. Loiseau, C. Culot, and L.P. Biró, Carbon 40 (2002) 1635-1648.

2 S. Amelinckx, A.A. Lucas, and Ph. Lambin, Rep. Progr. Phys. 62 (1999) 1471-1524.

3 V. Meunier and Ph. Lambin, Phys. Rev. Lett. 81 (1998) 5888-5891. 